\title{
A nonassociative schema theory of cognitive incompatibility
}

\author{
ASGHAR IRAN-NEJAD \\ University of Alabama, Tuscaloosa, Alabama
}

\begin{abstract}
Cognitive psychology has generated much research on similarity and the role it plays in the organization of knowledge in long-term memory. By comparison, little is known about the related concept of cognitive incompatibility. Building on the idea that mental schemas are incompatible to the extent they share elements, I discuss in this paper a nonconnectionist schema theory of incompatibility and compare it with the connectionist schema theory proposed by Rumelhart, Smolensky, McClelland, and Hinton (1986).
\end{abstract}

The term cognitive incompatibility, as it is used here, refers to the relationship that exists among mental structures that cannot be held in mind at the same time. One cannot, for instance, think of an individual as being both happy and sad or both a good Samaritan and a wolf in sheep's clothing. In this sense, cognitive incompatibility could be the basic ingredient that makes two concepts opposites. This, together with the sheer abundance of antonyms in natural languages, suggests that cognitive incompatibility may well be as fundamental to human thinking as similarity, which is the obvious basis for the universal occurrence of synonyms in natural languages. In this paper, the nature of incompatibility and its relation to similarity are examined.

\section{Biofunctional Schema Theory}

Unlike similarity, cognitive incompatibility has received little attention from researchers. One possible reason is that psychological theories have seldom provided the basis for a convincing way of thinking about incompatibility and its role in mental functioning. Modern cognitive psychology, for example, assumes that two structures are similar to the extent that they share elements, and different to the degree that they do not. What makes mental schemas incompatible remains unclear.

Iran-Nejad $(1980,1987)$ has proposed a biofunctional schema theory that implies, counterintuitively, that mental structures are incompatible to the extent that they are similar. Two aspects of this proposal combine to create an apparent paradox. On the one hand, we can say, for example, that "being cautious is like being careful," but we cannot say that "being happy is like being sad"

I wish to thank Abdollah Homaifar and Jim Nauta for their technical assistance, and Kevin Hughes for his comments. This research was supported in part by a National Academy of Education Spencer Fellowship and in part by a University of Alabama (RGC PM 1437) and a College of Education research grant. Send reprint requests to Asghar Iran-Nejad, Area of Behavioral Studies, Box 870231, Tuscaloosa, AL 35487-0231. or "being a good Samaritan is like being a wolf in sheep's clothing." However, the notion that incompatible structures are similar also makes sense. Black and white are opposite colors, but whatever they share to make them both colors makes them similar; two political parties may be thought of as opposites, but all the things that make them both political parties make them similar as well. To solve the paradox, one must specify (1) what it is that is similar or different about two incompatible structures, and (2) how these similarities or differences make schemas incompatible.

Biofunctional schema theory implies that incompatible structures are similar at the presubjective or submental level (Iran-Nejad \& Ortony, 1984). This basically means that they share elements to the extent that they are created by the same constellation of brain microsystems. The sharing of elements at the presubjective level explains why the similarity between incompatible structures is not as intuitively transparent as the similarity between two (subjectively) similar structures. At the subjective level, on the other hand, incompatible structures are different; which is why it makes no sense to say, for example, that being a good Samaritan is like being a wolf in sheep's clothing. Biofunctional schema theory implies that sharing elements at the submental level, together with being different at the mental level, is what makes schemas incompatible in the sense that they cannot be held in mind at the same time.

Sharing elements makes mental structures incompatible by making them automatically mutually exclusive (Diener \& Iran-Nejad, 1986). In other words, the reason why two schemas cannot be held in mind simultaneously is that they cannot coexist, subjectively or otherwise. They cannot coexist because the same set of microsystems is necessary for upholding both of them; and, as a result, at the moment these (shared) microsystems are busy creating one of the structures, the other cannot exist in the nervous system-just as two meetings cannot be held simultaneously when some of the same individuals must participate in both of them (Iran-Nejad, 1980). 


\section{Connectionist Schema Theory}

Automatic mutual exclusion (AME) may be compared with the mutual-inhibition idea that plays a central role in the connectionist schema theory proposed by Rumelhart, Smolensky, McClelland, and Hinton (1986). The connectionist mutual-inhibition (CMI) hypothesis implies that two mutually incompatible schemas are built on a tightly interconnected network of units consisting of two subnetworks, each with its own separate set of elements. The two schemas cannot be held in mind simultaneously because elements in one subnet are connected to those in the other by mutual inhibition paths. Rumelhart et al. used the Necker cube to show how mutual inhibition works in their model. I will use the same example to compare the AME and CMI hypotheses.

What makes the Necker cube relevant to the discussion of incompatibility is the well-known aspect that it permits two interpretations that cannot be held in mind simultaneously. The Necker cube drawing (Figure 1, bottom) can be seen either as a cube facing downward and to the left (dark cube on the left) or as one facing upward and to the right (dark cube on the right). To give each interpretation its own set of units, Rumelhart et al. (1986) postulated a 16-unit associative network consisting of two 8-unit subnets, one for each interpretation (top middle). To make the interpretations mutually incompatible, they connected the units in the two subnets with mutual inhibition paths.

Each of the 8 units in a subnet receives input from one of the 8 vertices in the cube drawing and represents a hypothesis about one of the two interpretations. For ex-

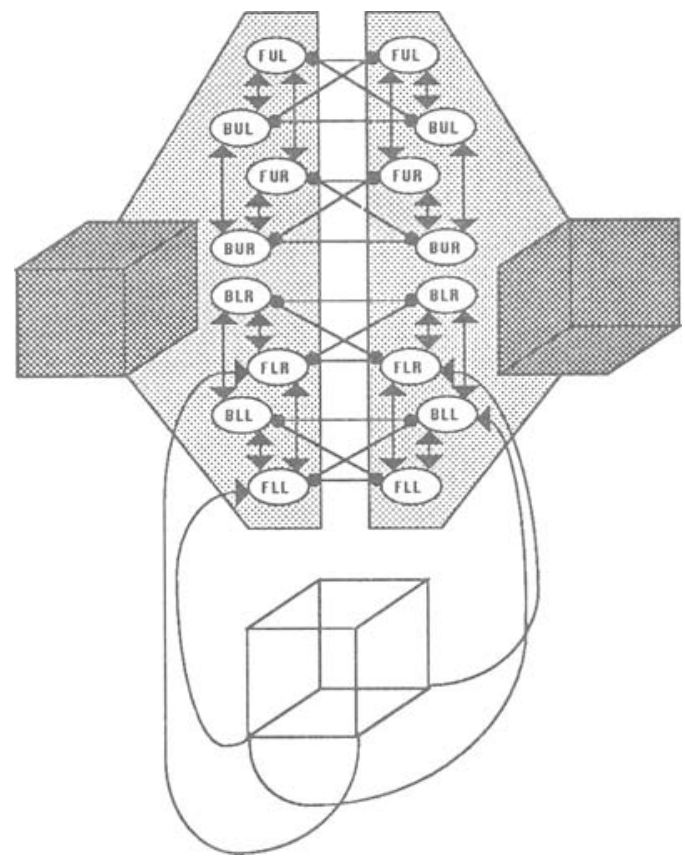

Figure 1. The Necker cube drawing (bottom) and the two subnetworks (upper middle) involved in its two interpretations (represented by dark cubes), based on connectionist schema theory. Only some of the network connections are shown. ample, the unit labeled FLR (for front, lower, right) in the subnet on the right receives input from the lower righthand vertex of the cube (one-way arrow) and represents a hypothesis consistent with the interpretation on the right: that the input-sending vertex is the front-lower-right corner of the cube.

Three assumptions govern the relationships in the network. Units in a subnet are connected to their neighbors within the same subnet by mutual excitation paths (vertical two-way arrows); units in one subnet are connected by mutual inhibition paths to those units in the other subnet that represent the same hypothesis (horizontal tentacles); and units in one subnet are connected by mutual inhibition paths to those units from the other subnet that receive input from the same cube vertex (diagonal tentacles). The CMI hypothesis of the connectionist model takes advantage of these assumptions to explain incompatibility by postulating separate associative subnetworks of elements connected by mutual inhibition paths.

\section{Biofunctional Schema Construction}

The AME hypothesis of biofunctional schema theory, on the other hand, assumes that mental structures are incompatible to the extent that they are (1) different at the mental level and (2) created by the same (submental) constellation of brain microsystems. Imagine that, under certain internal and external conditions, a distributed constellation of brain microsystems (Iran-Nejad, 1980, 1987) creates six TALK (tacit and live knowledge) components or mental sides (a front, a back, a top, a bottom, a right, and a left), and combines, uncombines, or recombines them to interpret, abandon an ongoing interpretation of, or reinterpret the Necker cube drawing. To show how AME works, one must then specify the (internal) conditions and processes that operate to construct two alternative interpretations using the same mental sides.

Some of the major conditions and processes involved are shown in Table 1 (Iran-Nejad, 1989). The table suggests that schema construction is a multisource process comprising three sources of control (external, active or monitor-dependent, and dynamic or monitor-independent) and five learning or constructive processes (attention, inquiry, closure, combination, and knowledge creation). The multisource perspective allows a plausible account of how the same TALK components, and presumably the same constellation of brain microsystems, can create and uphold two alternative interpretations of the Necker cube.

Briefly, when an individual finds the Necker cube in his or her visual field, the different sources of control begin to interact with attention and other learning processes to construct an interpretation of the drawing. For instance, if active internal control focuses attention-paying processes on a front, a top, and a right as visible sides (i.e., as sides under direct external control), the other learning processes that are high in active control (i.e., selfquestioning, prediction, and so on) will follow accordingly to set the stage for the interpretation that the drawing is a three-dimensional cube facing downward and to 
Table 1

Active and Dynamic Self-Regulation of Learning Processes

\begin{tabular}{llll}
\hline & \multicolumn{3}{c}{ Sources of Control } \\
\cline { 2 - 4 } Learning Processes & External & Active & Dynamic \\
\hline Attention & & & \\
1. Attention-catching & High & Low & Low \\
2. Attention-paying & Low & High & Low \\
3. Attention-holding & Low & Low & High \\
Inquiry & & & \\
1. Surprise & High & Low & Low \\
2. Self-questioning & Low & High & Low \\
3. Curiosity & Low & Low & High \\
Closure & & & \\
1. Orientation & High & Low & Low \\
2. Prediction & Low & High & Low \\
3. Postdiction & Low & Low & High \\
Combination & & & \\
1. Independent & High & Low & Low \\
2. Sequential & Low & High & Low \\
3. Simultaneous & Low & Low & High \\
Knowledge Creation & & & \\
1. Categorical & High & Low & Low \\
2. Propositional & Low & High & Low \\
3. Thematic & Low & Low & High \\
\hline
\end{tabular}

From "Associative and nonassociative schema theories of learning," by A. Iran-Nejad, 1989, Bulletin of the Psychonomic Society, 27, 1-4. Copyright 1988 by Psychonomic Society, Inc. Reprinted by permission.

the left. Active internal control alone, however, will not be enough; and dynamic (or monitor-independent) internal sources must also play their indispensible role. When this happens, learning processes that are high in dynamic control (e.g., attention-holding, curiosity, and so on) will join in to contribute to the sequential and simultaneous process of schema construction. In this way, the different sources of control and learning processes can construct and uphold the interpretation shown on the left-hand side of Figure 1.

As an example, consider the contribution of active inquiry (or self-questioning) processes. Upon seeing the Necker cube drawing, the individual might ask himself or herself questions about what the drawing represents. Similarly, at some point during the construction process, when the drawing is about to be seen as a threedimensional cube, the individual might predict that the cube also has a back, a bottom, and a left side that are invisible. As an example of the operation of dynamic inquiry processes, imagine a person looking at an actual cube, instead of a drawing, with the front-top-right pattern of visibility. He or she might experience the urge of curiosity to check to see if the three invisible sides are indeed there as well.

Thus, the biofunctional model specifies some of the major external/internal conditions determining the interpretation of the Necker cube drawing as a cube facing downward and to the left or as one facing upward and to the right. The first interpretation is constructed if a front, a top, and a right are focused as the visible sides, and a back, a left, and a bottom as invisible sides. The second interpretation is constructed if a front, a bottom, and a left are focused as the visible sides, and a back, a top, and a right as invisible sides. However, regardless of which sides are focused as visible or invisible, given the Necker cube drawing as the stimulus, all six mental sides must participate in the construction of both interpretations, which is why the two interpretations cannot be held in mind simultaneously.

The biofunctional model also shows exactly what must change in order for the perceiver to switch back and forth between interpretations: the selected pattern of visibility must change, and the sides representing the front and the back must reverse roles. Everything else will stay the same. The model, therefore, explains how two incompatible mental structures can be constructed out of a minimum number of mental units. It also specifies the minimum amount of change that must occur for an observer to shift his or her interpretation from one structure to another.

\section{The Incompatibility-Similarity Relationship}

The idea that sharing elements is the basis of incompatibility implies that cognitive similarity and incompatibility are related in a fundamental way; but it also tends to blur the obvious distinction between them. Exactly how is the relationship between two incompatible structures different from the relationship between two similar structures? Broadly speaking, if the biofunctional model is correct, mental structures are similar or incompatible because they share elements. In both cases, the components of one structure must participate in the creation and upholding of another. We must, therefore, try to distinguish between similarity and incompatibility, not at the level of elements, but at the level of the whole. Similarity and incompatibility are emergent characteristics, and, contrary to the assumptions upon which connectionism is based, they cannot be represented in the microstructure of the mind (or the brain).

A more precise view of the similarity-incompatibility relationship can be given in terms of the informationcreation processes shown in Table 1. Iran-Nejad (1980, 1987) used a light-bulb constellation analogy to show how information creation might happen. Imagine a system consisting of a vast number of color-coded light bulbs. In such a system, constellations of burning light bulbs can create patterns of light. Furthermore, either burning light bulbs can stay on constantly, or, if so designed, they can generate blinks. Similarly, according to the biofunctional model, constellations of brain microsystems can generate knowledge structures, as opposed to storing them. Furthermore, brain microsystems can engage in two qualitatively different types of activity and thereby create two qualitatively different types of knowledge. One kind, called thematic knowledge, can result from the staying-on activity of large constellations of brain microsystems whose elements are distributed throughout the entire nervous systems. Thematic knowledge can emerge out of the combined, simultaneous, and ongoing activities of these microsystems and can represent whole-level knowledge. The second type, categorical knowledge, is unit-level knowledge and can be created by momentary blinks, as opposed to the staying- 
Table 2

Elemental and Thematic Determinants of Between-Schema Relations

\begin{tabular}{lll} 
& \multicolumn{2}{c}{ Theme } \\
\cline { 2 - 3 } Elements & \multicolumn{1}{c}{ Same } & \multicolumn{1}{c}{ Different } \\
\hline Shared & Similar & Incompatible \\
Not shared & Metaphor & Different \\
\hline
\end{tabular}

From "Associative and nonassociative schema theories of learning," by A. Iran-Nejad, 1989, Bulletin of the Psychonomic Society, 27, 1-4. Copyright 1988 by Psychonomic Society, Inc. Reprinted by permission.

on activity produced by subconstellations of brain microsystems. The third type, propositional knowledge, is actually not a new type, in the sense of representing a qualitatively different kind of brain activity. Rather, it results from the sequential generation of categorical knowledge.

Since unit-level knowledge categories are created by a momentary or short-term ("blinking") process, and since they directly represent only the sequential portion of the ongoing schema and not necessarily the entire mass, they cannot adequately represent all the components that two similar or incompatible schemas can share. Rather, it is theoretically more meaningful to think of elementsharing at the level of brain microsystems; only these microsystems, whose staying-on activity upholds the holistic aspect of the ongoing mental schema, can represent adequately, at least in principle, the entire set of components involved in an ongoing mental schema. Similarly, only whole-level or thematic knowledge, and not necessarily categorical or propositional knowledge, is likely to represent adequately the characteristics that might distinguish between two incompatible schemas.

Having fixed element-sharing at the level of brain microsystems and whole-level differences at the level of thematic knowledge, we can turn to Table 2 to examine the nature of the similarity-incompatibility relation. Two schemas are similar to the extent that they share elements and have the same theme. They are incompatible to the degree that they share elements and have different themes (Iran-Nejad, 1989).

\section{Summary and Conclusions}

In this paper, it has been shown that the sharing of elements can be the basis of incompatibility. Although this notion runs counter to the connectionist perspective and appears to be counterintuitive, it is a possible way of thinking about incompatibility. Sharing elements leads to automatic mutual exclusion, eliminating the need for the postulation of separate associative subnetworks connected by mutual inhibition paths. The mutual inhibition hypothe- sis represents incompatibility at the level of the microstructure of cognition. Many psychologists agree that attempts to represent aspects of cognition in associative nodes and connections are likely to face the insurmountable problem that the whole is more than the sum of its parts. Nevertheless, in its most sophisticated form yet, connectionism is currently gaining momentum and prestige. In this paper, it has been shown that cognitive incompatibility can be represented neither at the level of elements nor at the level of holistic structures. Rather, it emerges out of the interaction between component-level and whole-level aspects of mental functioning.

The hypothesis that two schemas are incompatible to the extent that they share components implies that generalization must occur not just across two similar schemas, but between two incompatible schemas as well. In effect, generalization across incompatible structures means abandoning an ongoing interpretation without abandoning the elements that are upholding it, and using these elements, perhaps together with some new ones, to construct a different interpretation. Table 2 suggests that generalization across two similar schemas does not involve a thematic change, but generalization across incompatible schemas does. It follows that genuine schema learning occurs only when there is generalization from one ongoing mental structure to a new mental structure incompatible with it; it is only then that a truly different cognitive structure is constructed out of the elements of an old schema.

\section{REFERENCES}

Diener, E., \& IRAN-NeJAD, A. (1986). The relationship in experience between various types of affect. Journal of Personality \& Social Psychology, 50, 1031-1038.

IRAN-NEJAD, A. (1980, February). The schema: A structural or a functional pattern (Technical Report No. 159). Urbana: University of Illinois, Center for Study of Reading.

IRAN-NEJAD, A. (1987). The schema: A long-term memory structure or a transient functional pattern. In R. J. Tierney, P. Anders, \& N. Mitchell (Eds.), Understanding readers' understanding (pp. 109127). Hillsdale, NJ: Erlbaum.

IRAN-NEJAD, A. (1989). Associative and nonassociative schema theories of learning. Bulletin of the Psychonomic Society, 27, 1-4.

IrAN-NEJAD, A., \& OrTONY, A. (1984). A biofunctional model of distributed mental content, mental structures, awareness, and attention. Journal of Mind \& Behavior, 5, 171-210.

Rumelhart, D. E., Smolensky, P., McClelland, J. L., \& HiNTon, G. E. (1986). Schemata and sequential thought processes in PDP models. In J. L. McClelland, D. E. Rumelhart, \& the PDP Research Group (Eds.), Parallel distributed processing (Vol. 2, pp. 7-57). Cambridge, MA: MIT Press.

(Manuscript received February 16, 1989.) 